\title{
Magnetic Wall Climbing Robot for Thin Surfaces with Specific Obstacles
}

\author{
W. Fischer ${ }^{1}$, F. Tâche ${ }^{2}$, and R. Siegwart ${ }^{3}$ \\ ${ }^{1}$ Swiss Federal Institute of Technology Zürich wfischer@ethz.ch \\ ${ }^{2}$ Swiss Federal Institute of Technology Zürich ftachedethz . ch \\ ${ }^{3}$ Swiss Federal Institute of Technology Zürich rsiegwart@ethz . ch
}

Summary. This paper describes a novel solution to a mobile climbing robot on magnetic wheels, designed for inspecting the interior surfaces in gas tanks made out of thin metal sheets. These surfaces were inaccessible by previous climbing robots due to the following restrictions:

1. Ridges, where the magnetic force decreases to almost zero

2. Angular transitions between the surfaces $\left(135^{\circ}\right)$

3. Thin metal sheets that cannot provide high magnetic forces

The main optimization criterion for this robot was to design it as light as possible, as the surface was also considered to be very fragile. As the here described type of application is very special and was not examined much in previous publications, this work also stresses on the early analysis phase. This phase mainly consists of tests to optimize magnetic wheels for thin surfaces and mechanical calculations for robots on magnetic wheels. The chosen concept is described in detail, explaining how the robot moves around and passes the obstacles. The analysis of the most critical cases is presented, as well as some details about magnetic wheels and actuators.

\section{Introduction}

The robot described here was designed for inspecting gas tanks that are made out of thin metal sheets and are installed in huge ships. From time to time, they have to be analyzed for leaks, especially at the welds. For doing this, helium is injected in the surrounding structure from outside. A sensor that can detect this helium then is moved to all places inside the tank to find the position of the leak. Until now, this sensor was carried by a balloon that was operated manually, using some ropes. As this method was very slow and imprecise, a better inspection system, preferably consisting of a climbing robot on magnetic wheels, had to be developed. As the environment cannot support much force, the main goal was to make this robot as light as possible. To simplify the control and increase the reliability, another method was using only few actuators. To ensure a correct functionality, the most critical risks were analyzed. This analysis does not only incorporate the possibility of some components within the robot breaking down. It also accounts for the risks of plastically deforming the environment, falling or slipping. 
The work is structured into seven sections: Section 2 provides a short overview on the state of the art considering mainly climbing robots on magnetic wheels and their mechanisms to pass obstacles. Section 3 explains the application and deduces the most critical requirements. In Section 4 the first tests and analyses are presented that were necessary to obtain the best concept in an effective way. Sections 5 and 6 describe the chosen concept and explain how it works in the most critical cases. The last section provides a short summary, together with an outlook on future versions of the robot.

\section{State of the art}

The robot analyzed here is a mobile climbing robot that uses magnetic wheels for also moving on vertical walls and on the ceiling. In order to pass difficult obstacles, it uses active elements within the structure. For better structuring, this section is divided into the following 3 fields of technology.

\subsection{Mobile climbing robots}

Mobile climbing robots are used in various applications - not only for nondestructive testing, but also for cleaning, repairing and other tasks. As the applications are very different, there exist many types of robots. The two main criteria to distinguish these types are the adhesion mechanism and the locomotion principle. According to [1], the most common adhesion mechanisms are magnetism [2]-[12], vacuum suction [13]-[15], specific attachment devices such as rails [17] or pegs and grippers/clamps [16]. The locomotion can be based either on wheels [2]-[8], tracks [11], legs [9] or arms. For both profiting from the simple control of wheeled locomotion and the high obstacle passing capabilities of legged locomotion, some robots use active elements within the structure ([8], [15], and [19]). As the here described robot uses magnetic wheels and a structure with active elements, the following descriptions mainly stress on this type.

\subsection{Climbing robots on magnetic wheels}

The simplest structure for a climbing robot is a vehicle on two (like the "Magnebots" [2]) or more magnetic wheels. These wheels increase the normal force to the ground and thus allow for being more independent from the direction of gravity. The most common structure of such wheels is described in U.S. Patent 3,690,393 [3] and drawn in Fig. 3: A cylindrical magnet in the middle; two plates out of magnetic steel and of slightly bigger diameter on both sides (to better conduct the magnetic force into the surface); and a thin layer of rubber around the steel plates (to increase the friction to the ground from $\mu=0.3-0.5$ to $\mu=0.5-0.8$ ). Sometimes the wheels are also equipped with some special mechanisms that facilitate passing angular transitions. These mechanisms can either be passive [6] or active [7]. The most common applications for these robots are non-destructive testing on the outside surface of storage tanks [4], [5] and general transport tasks [2]. 


\subsection{Active elements in the structure}

Active elements in the structure are often used in wheeled robots to profit from both the advantages of wheeled and legged locomotion - high speed and simple control (wheels) combined with an extended mobility (legs). Examples for this type are the "Alicia3" climbing robot with suction pumps [15] or the "Octopus" rough-terrain robot [19]. In [8], the "Pipe Inspection Robot (PIR)" is described. This robot uses 3 pairs of magnetic wheels that can be lifted with linear actuators in order to pass obstacles on the outside surfaces of pipes. Its structure has some similarities to our robot, but needs more actuators (26 in the latest version that is also able to turn) and is not able to move on very thin surfaces where the magnetic force gets saturated.

\section{Application and requirements}

As already pointed out in the introduction, the goal is to develop a climbing robot that can bring a sensor module (approximately $1 \mathrm{~kg}$ ) to every point in these specific gas tanks (Fig. 1) - an extremely difficult environment where it was impossible to go with previous climbing robots due to the following difficulties:

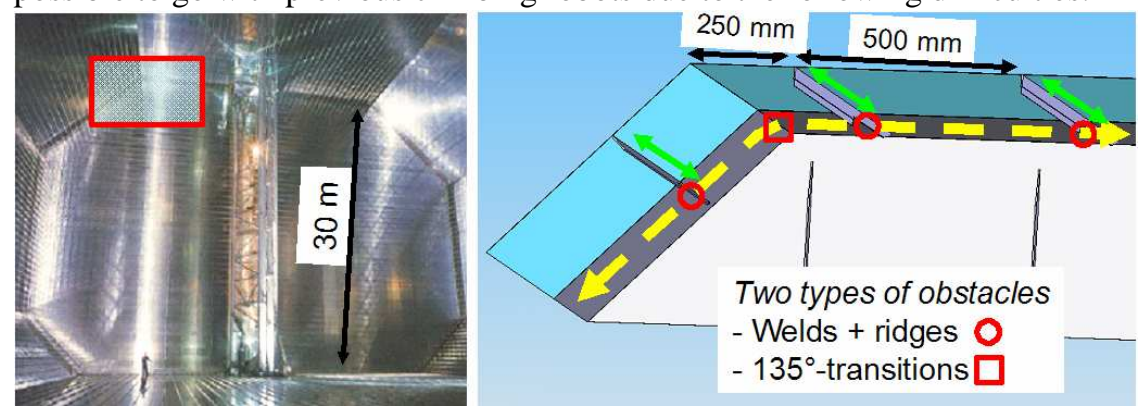

Fig. 1. The environment to inspect

\subsection{Obstacles: Ridges and angular $135^{\circ}$-transitions}

The most difficult obstacles in this environment are the ridges. Their height ranges up to $40 \mathrm{~mm}$. Whereas just rolling onto them would not be difficult with big enough wheels, the most critical problem is that the magnetic force on top of these ridges decreases to less than $15 \%$ of the original value. Thus, when rolling on a vertical wall or on the ceiling, the robot would slip or fall down if the attraction force is not compensated by another wheel that stays on the ground.

The other type of obstacle is the angular $135^{\circ}$-transition between two surfaces. As the surface is not rounded, the wheels get in contact at two points. When moving away from these points whilst entering the new surface, the robot needs a lot more traction than for just climbing a vertical wall. 

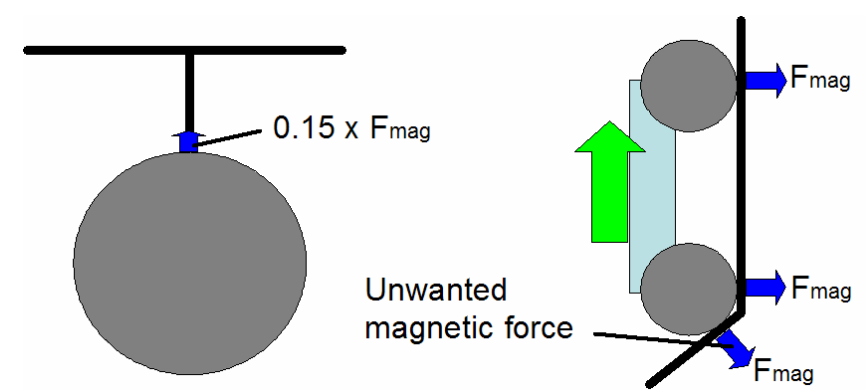

Fig. 2. Obstacles: force decrease on ridges (left); unwanted forces at angular $135^{\circ}$ transitions (right) Both obstacles only are encountered on paths perpendicular to the ridges (Fig. 1, dashed yellow line). On horizontal paths (Fig. 1, green line) there are no obstacles. The distance between the ridges is always $500 \mathrm{~mm}$, between the last ridges and the $135^{\circ}$ transition it is only $250 \mathrm{~mm}$. This distance limits the maneuvering space of the robot.

\subsection{Reduced magnetic force on thin sheet material}

The fact that the sheets are very thin $(1.5 \mathrm{~mm})$ does not only cause problems in terms of environment fragility. It also limits the magnetic forces:
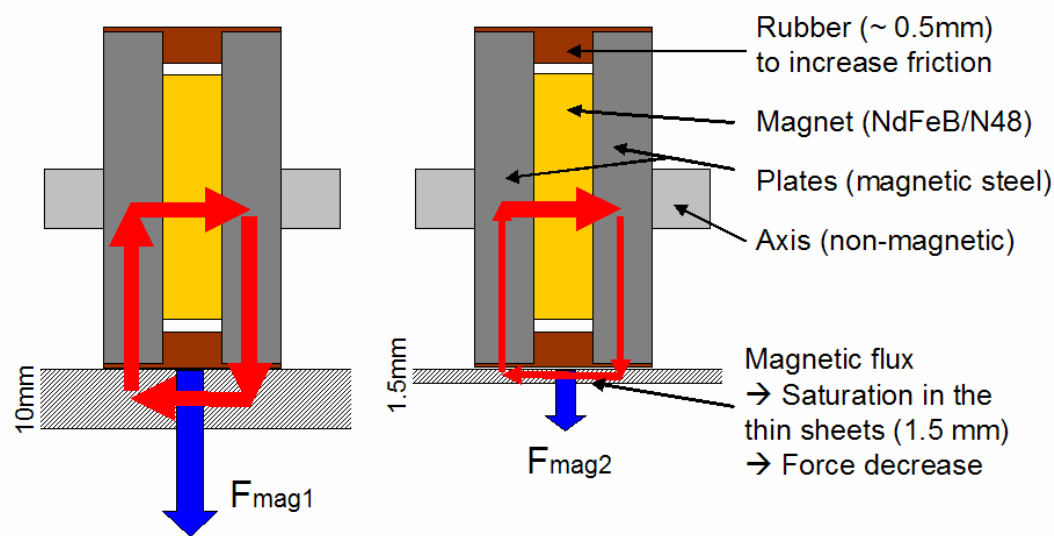

Fig. 3. Simple magnetic wheel [3] and the force decrease on thin ground

In order to result in high attraction forces, the magnetic flux has to go mainly through the metallic surface of the environment, as the magnetic resistance there is lower than in air. If this metallic surface is very thin, the magnetic flux becomes saturated there and the force is then limited. In our case we measured approximately $90 \mathrm{~N}$ for an $\emptyset 60 \mathrm{~mm}$-wheel that could hold $200 \mathrm{~N}$ on $10 \mathrm{~mm}$ thick metal plates. For this reason, preventing the robot from slipping or falling down in the most difficult situations was crucial.

\subsection{General goals}

Apart from just moving around in this difficult environment, the goals were to achieve a horizontal speed of at least $5 \mathrm{~m} / \mathrm{min}$, to keep the mass below $10 \mathrm{~kg}$ and to keep the whole system as simple and reliable as possible. 


\section{First analysis and wheel optimization}

At first, the prior idea - the fact that a robot on magnetic wheels really is the best concept for this application - had to be verified. When analyzing alternatives, all of them did not seem as promising: A robot with suction cups and a pump would be heavy, complex and not safe in case of power interruption. A robot on clamps or on magnetic legs would not be fast enough to achieve the required minimum speed $(5 \mathrm{~m} / \mathrm{min})$. Installing rails on the whole surface would be too much effort. Robots on magnetic tracks did not seem promising either; as such tracks would be difficult to turn and be even worse to pass the ridges. Finally, a locomotion based on magnetic wheels seemed to be the best possibility. For better estimating the necessary properties of such a robot, some more analysis in this field of technology was necessary, consisting of some tests, calculations and simulations.

\subsection{Tests and improvement of the magnetic wheels}

For testing the magnetic force, we used a tension spring balance and pulled the wheels off from different types of surfaces. Whereas on thick plates $(10 \mathrm{~mm})$, $200 \mathrm{~N}$ were measured, on the thin sheets $(1.5 \mathrm{~mm})$ only $90 \mathrm{~N}$ were possible.

For obtaining enough force on this thin surface also, two magnetic wheels were set in parallel. For making the whole structure lighter, the wheel plates were made in a conic shape. This structure saves about $30 \%$ of mass without decreasing the magnetic force. For increasing the friction and protecting the environment, all wheel plates are covered with a thin rubber.

\subsection{Analysis of the ridges}

Together with the above tests, we did also analyze the behavior when rolling over the ridges. As already pointed out in the previous section (Fig. 2) the magnetic force thereby decreases to only $15 \%$. Thus, in order to pass these ridges, an extra mechanism is necessary that can compensate for this force decrease. Among various possibilities for obtaining this goal, the one with two nonmotorized extra wheels outside the central body and driven by linear actuators was considered as the best. How this mechanism passes the ridges is described in section VI.

\subsection{Mechanical calculation $135^{\circ}$-transitions, roofs and verti- cal walls}

The $135^{\circ}$-transitions were calculated for all 8 cases if inclination and both directions each. As the speed is supposed to be very slow, these calculations could be done with a static model. Estimating a mass of $10 \mathrm{~kg}$ and realistic values for the geometry, we estimated a required friction coefficient of $\mu$ required $=0.37$ and a necessary torque of $3.5 \mathrm{Nm}$ in each wheel pair. 
Also, the robot on the roof and when passing the ridges on vertical walls (with traction on only one motorized wheel pair, Fig. 5) was analyzed. These two cases resulted to be less critical than the $135^{\circ}$-transitions: The necessary motor torque for passing ridges on a vertical wall is only about $50 \%$ of the maximum value, and also the required friction coefficient $\mu$ required (risk against slipping) was calculated to be less critical.

The risk against falling off the roof was not seen as critical either, as for passing ridges and $135^{\circ}$-transitions, the magnetic force has to be strong enough to prevent slipping.

\section{The chosen concept}

Among many promising concepts, the following one (Fig. 4) was selected to be the best, considering simplicity, mass, security against slipping and maneuvering space. It consists of 4 independently motorized wheel units, each of them being equipped with a steering motor, such that it can turn on spot. The wheels are designed as described in the last section - with conic shaped plates and always two in parallel.

For passing the ridges, two lifter units are situated outside the central body. These lifter units use linear actuators consisting of a spindle inside a stepper motor. This type of actuator has a much better force/mass ratio than other actuators of the same size $(300 \mathrm{~N}$ at only $300 \mathrm{~g})$ and thus was preferred. To guide the movement, linear slide bearings are used. The total mass was estimated to $10 \mathrm{~kg}$ in the CAD software. This value will perhaps increase a little bit, when all sensors and motor controllers will be included. The magnetic force was measured to $170 \mathrm{~N}$ for each wheel unit and to $2 \times 90 \mathrm{~N}$ for each small wheel unit on the lifter. The total size amounts to $200 \times 200 \times 300 \mathrm{~mm}^{3}$, approximately.

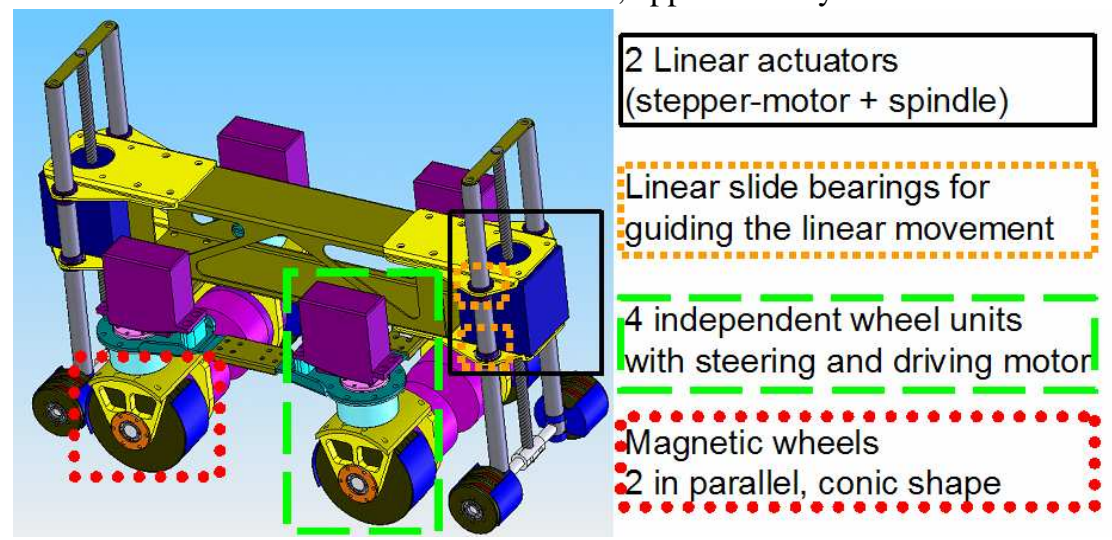

Fig. 4. Overview on the chosen concept 


\section{Behavior in the most critical cases}

\subsection{Ridges}

For passing the ridges, the main wheels can be lifted off the ground. During this lift-off, the missing attraction force is taken over by the non-motorized extra wheels (Fig. 5). For saving mass, space and reducing the force in the linear actuators, these extra wheels are smaller and less strong than the main wheels $($ FmagE $=2 \times 90 \mathrm{~N}$ instead of $2 \times 170 \mathrm{~N})$. As they only have to keep the robot on the wall but do not have to transmit traction forces, this force was calculated to be enough.

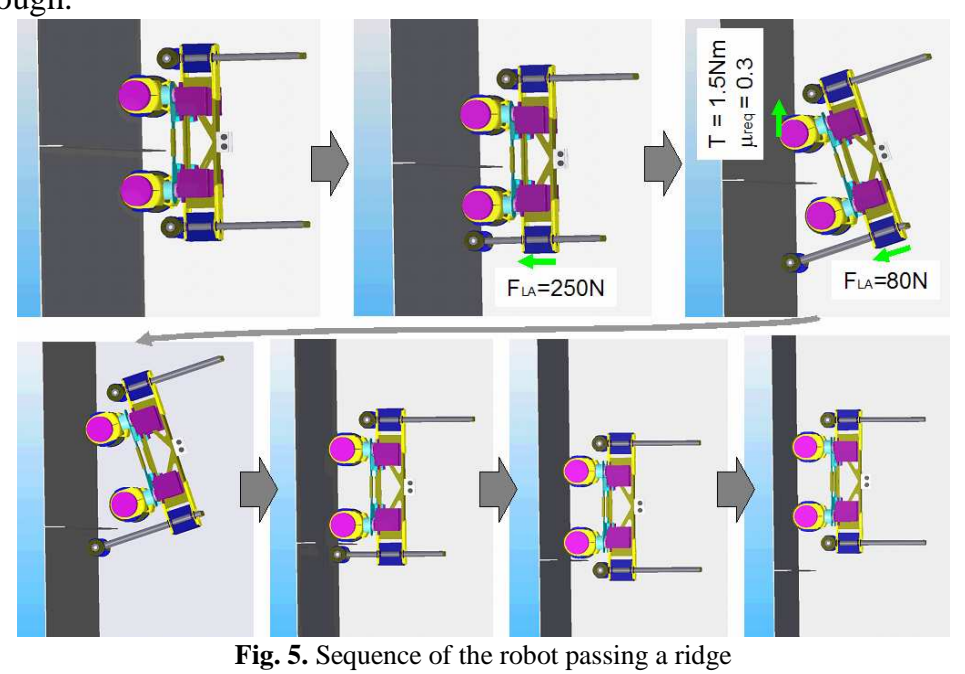

With realistic values for geometry, mass and magnetic force $(\mathrm{m}=10 \mathrm{~kg}$ and Fmag $=170 \mathrm{~N}$ ) the maximum required force in the linear actuator was calculated to $250 \mathrm{~N}$ (allowed value: $300 \mathrm{~N}$ ). This force occurs, when the main wheel units are detached from the ground. The required maximal force during the rest of the movement (originating from the mass and the friction losses in the linear slide bearings) was also calculated and is much smaller $(80 \mathrm{~N})$.

The required torque in each drive motor was calculated to $1.5 \mathrm{Nm}$. The required friction coefficient was calculated to $\mu$ req $=0.3$ (measured value: $\mu \geq 0.5$ ). Thus the robot is safe against slipping.

\subsection{Angular $135^{\circ}$-transitions}

For passing the $135^{\circ}$-transitions, the robot has to roll over the obstacle with a torque distribution that is optimized to have the same required friction coefficient in all contact points (Fig. 6). In the worst case the required motor torque has to be $1.5 \mathrm{Nm}$ in the front wheels and $3.5 \mathrm{Nm}$ in the rear ones. The maximum required friction coefficient is then $\mu$ req $=0.37$. These values for friction and motor torque are higher than for the previous case (ridges), but still acceptable (maximum allowed motor torque: $4.5 \mathrm{Nm}$; measured friction coefficient: $\mu \geq 0.5$ ). 


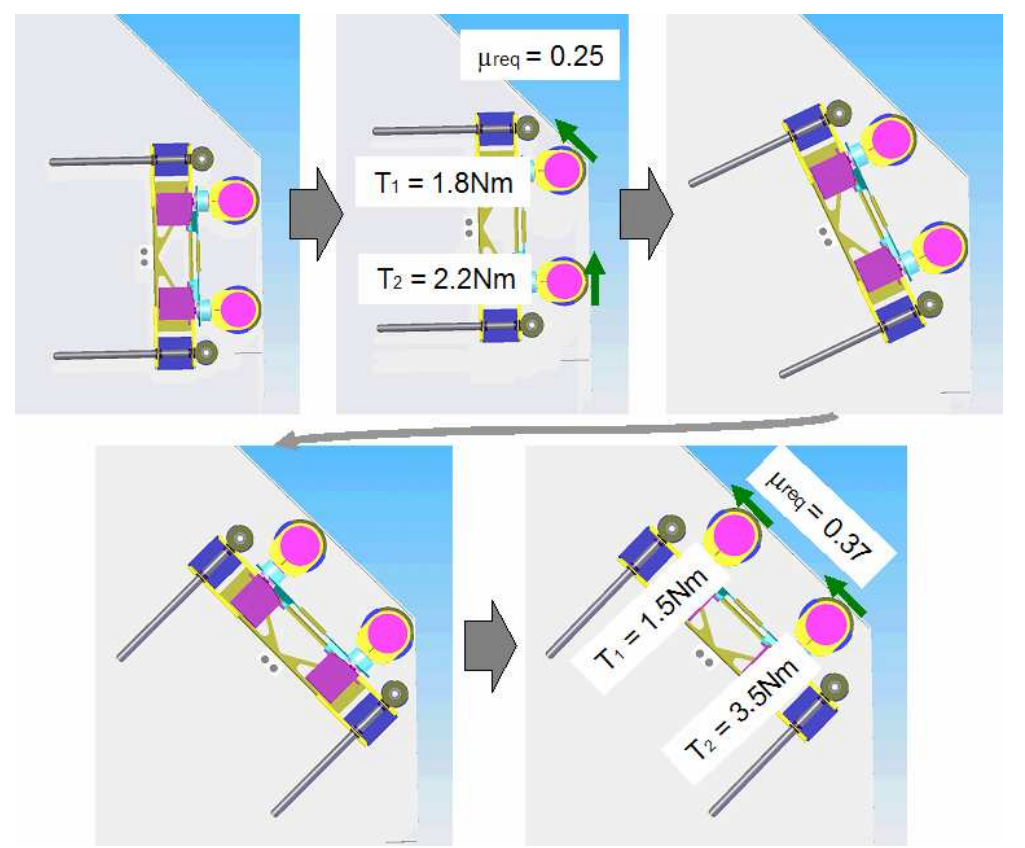

Fig. 6. The robot passing a $135^{\circ}$-transition (optimized torque distribution)

\subsection{Turning}

For switching from the vertical path to the horizontal one, the wheels turn on spot (Fig. 7). Each wheel is actuated by one steering motor. Here, assuming a friction coefficient of $\mu=0.8$ (estimated maximum value) and a distance between the two wheels in each pair of $d=20 \mathrm{~mm}$, the necessary steering torque resolves to $2.7 \mathrm{Nm}$ in each steering axis. As the planned actuators (servo motors) can provide $3.5 \mathrm{Nm}$, turning the wheels is not a problem either.

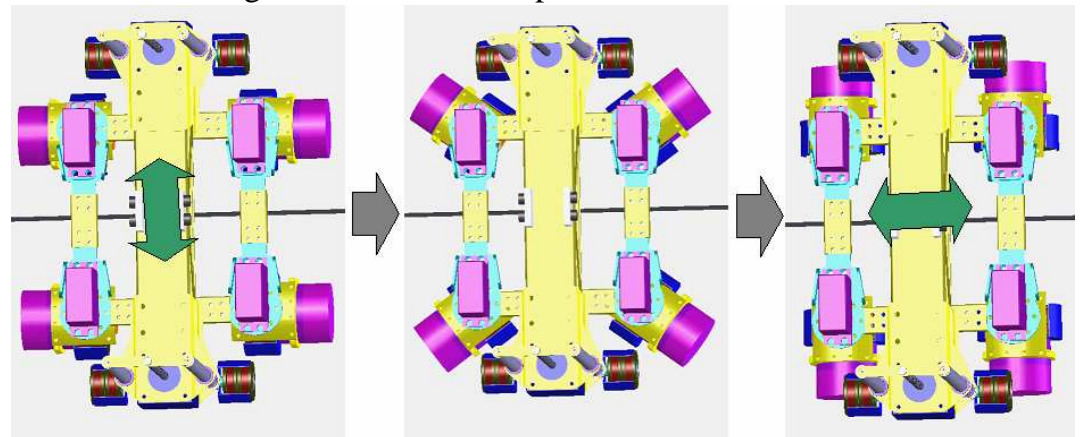

Fig. 7. The robot switching from vertical to horizontal paths

The possibility to turn the whole robot instead of only the wheels was analyzed as well. In this configuration, fewer actuators would have been needed. However, it could not be used, as the allowed maneuvering space between the ridges and the $135^{\circ}$-transitions was not enough to perform this movement (Fig. 1). 


\section{Conclusion and outlook}

In this work, a novel solution to a magnetic wheeled climbing robot for thin and fragile surfaces was developed, with the capability to work in all inclinations of the surface, to pass two types of difficult obstacles and to turn on spot. Due to optimizations in structure and components, its mass will be around $10 \mathrm{~kg}$, which also allows for going in fragile environments without destroying them. The size will be around $200 \times 200 \times 300 \mathrm{~mm}^{3}$ - small enough to fit well in the environment, but big enough to pass ridges of $40 \mathrm{~mm}$ height. As only 10 actuators are used (instead of 26 in the most similar robot that was found in the state of the art [7]), cost, complexity and reliability will be limited to a reasonable range.

Due to measurements on already built parts (magnetic wheels and actuators), calculations and simulations, we could prove that all actuators will be strong enough and that the robot will not slip or lose contact at any time. Even for the most critical case - the risk of slipping in the $135^{\circ}$-transitions - the calculated required friction coefficient ( $\mu$ required $=0.37$ ) lies significantly below the minimum value that was measured with a real wheel ( $\mu$ measured $\geq 0.5$ ). Thus, it is assured that the concept will work well in the specified application - an environment that was impossible to be accessed by previous climbing robots.

However, when our industrial partners analyzed again the environment in these gas tanks, it resulted that the specifications that formed the base for developing this concept were not complete: On the largest part of the surface - except the last $500 \mathrm{~mm}$ up to the neighboring walls (Fig. 1 grey area) - the sheet thickness is only $0.7 \mathrm{~mm}$ instead of $1.5 \mathrm{~mm}$, resulting in a significant loss of magnetic force there. After doing more detailed stress tests, it also resulted that on these sheets the allowed mass should be only $5 \mathrm{~kg}$ - instead of $10 \mathrm{~kg}$ as it was specified before. However, on the thick surface even $20 \mathrm{~kg}$ could be allowed. With these changed specifications, a safe operation could not be assured any more with the design described above.

Thus, for dealing with these changed specifications, the whole concept had to be adapted. The advanced design has already been started and will be realized soon. It is based on the same obstacle passing mechanism, but uses the additional idea to separate the whole system into two robots instead of going everywhere with only one big and complex one:

The bigger robot passes the obstacles and always stays on the thick surface where the magnetic attraction is strong and $20 \mathrm{~kg}$ of robot mass is allowed. For the horizontal paths (no obstacles, but only $5 \mathrm{~kg}$ allowed), it sends out a smaller and simpler robot with only one degree of freedom. For the bigger one, the same obstacle-passing mechanism will be reused, on a structure that is optimized to the now changed requirements: No turning, but carrying the smaller robot. 


\section{References}

1. Balaguer, C.; Virk, G.; Armada, M., "Robot applications against gravity", Robotics \& Automation Magazine, IEEE, Volume 13, Issue 1, March 2006, Pages: 5- 6

2. Slocum, H.; Awtar, S.; Hart, J., "Magnebots: Magnetic Wheels Based Overhead Transportation Concept", Conference paper from IFAC Mechatronics 2002 (Berkeley, Dec/2002)

3. Guy, W., "Magnetic Wheel", U.S. Patent 3,690,393, September 1972

4. Sogi, T.; Kawaguchi, Y.; Morisaki, H.; Ohkawa, K.; Kai, N.; Hayakawa, H., "Inspection robot for spherical storage tanks", Industrial Electronics Society, 2000. IECON 2000. 26th Annual Confjerence of the IEEE, Volume 1, 22-28 Oct. 2000 Page(s):393 - 398 vol.1

5. Jireh Industries Ltd.; "Tripod - a Revolution in Remote Transportation"; Industrial product; www.jireh-industries.com/

6. Kawaguchi, Y.; Yoshida, I.; Kurumatani, H.; Kikuta, T.; Yamada, Y.; 'Internal pipe inspection robot", Robotics and Automation, 1995. Proceedings., 1995 IEEE International Conference on Volume 1, 21-27 May 1995, Page(s):857 - 862 vol.1 0

7. Tâche, F.; Fischer, W.; Siegwart, R.; “Adapted Magnetic Wheels for Robots Inspecting Narrow Pipes with Complex Shaped Structures“, submitted for publication at IEEE/ASME International Conference on Advanced Intelligent Mechatronics, September 4-7, 2007

8. Yukawa, T.; Okano, H.; Komatsubara, S.; "Mechanisms for the movement of piping inspection robot with magnetic elements", Proceedings of the IASTED International Conference ROBOTICS AND APPLICATIONS, 2005, Cambridge, USA525390

9. Grieco, J.C.; Prieto, M.; Armada, M.; Gonzalez de Santos, P.; "A six-legged climbing robot for high payloads", Proceedings of the 1998 IEEE International Conference on Control Applications, Volume 1, 1-4 Sept. 1998 Page(s):446 - 450 vol.1

10. Hirose, S.; Tsutsumitake, H.; "Disk Rover: A Wall-climbing Robot Using Permanent Magnetic Discs", Proceedings of the 1992 lEEE/RSJ International Conference on Intelligent Robots and Systems, Volume 3, July 7-10,1992 Page(s):2074 - 2079

11. Weimin Shen; Gu, J.; Yanjun Shen; "Proposed wall climbing robot with permanent magnetic tracks for inspecting oil tanks", Mechatronics and Automation, 2005 IEEE International Conference, Volume 4, 29 July-1 Aug. 2005 Page(s):2072 - 2077 Vol. 4

12. Kitai, S.; Tsuru, K.; Hirose, S.; "The proposal of swarm type wall climbing robot system "Anchor Climber" - design and examination of the adhering mobile unit", 2005 IEEE/RSJ International Conference on Intelligent Robots and Systems, 2-6 Aug. 2005 Page(s):475 - 480

13. Jizhong Xiao; Sadegh, A.; Elliott, M.; Calle, A.; Persad, A.; Ho Ming Chiu; "Design of Mobile Robots with Wall Climbing Capability (overlook on pneumatic robots)", Advanced Intelligent Mechatronics. Proceedings, 2005 IEEE/ASME International Conference on, $2005 \mathrm{~Pa}-$ ge(s):438 - 443

14. Pack, R.T.; Christopher, J.L., Jr.; Kawamura, K.; "A Rubbertuator-based structure-climbing inspection robot", Robotics and Automation, 1997. Proceedings., 1997 IEEE International Conference on, Volume 3, 20-25 April 1997 Page(s):1869 - 1874 vol.3

15. Longo, D.; Muscato, G. , "Alicia/sup 3/ climbing robot: a three-module robot for automatic wall inspection”, Robotics \& Automation Magazine, IEEE, Volume 13, Issue 1, March 2006 , Page(s): 42- 50

16. Aracil, R.; Saltaren, R.J.; Reinoso, O., “A climbing parallel robot: a robot to climb along tubular and metallic structures", Robotics \& Automation Magazine, Volume 13, Issue 1, 2006, Page(s): 16- 22

17. Yamamoto, S.; "Development of inspection robot for nuclear power plant", Proceedings., 1992 IEEE International Conference on Robotics and Automation, 12-14 May 1992 Page(s): 1559 - 1566 vol.2

18. Balaguer, C.; Gimenez, A.; Huete, A.J.; Sabatini, A.M.; Topping, M.; Bolmsjo, G., "The MATS robot: service climbing robot for personal assistance" , Robotics \& Automation Magazine, IEEE, Volume 13, Issue 1, March 2006, Page(s): 51- 58

19. Lauria, M., Piguet, Y. and Siegwart, R. (2002) Octopus - An Autonomous Wheeled Climbing Robot. In Proceedings of the Fifth International Conference on Climbing and Walking Robots. 